\title{
Ultrasound and magnetic resonance molecular imaging of atherosclerotic neovasculature with perfluorocarbon magnetic nanocapsules targeted against vascular endothelial growth factor receptor 2 in rats
}

\author{
HUA CHEN $^{1,2}$, LIANGLONG CHEN ${ }^{1,2}$, RONGXI LIANG ${ }^{3}$ and JIN WEI ${ }^{4}$ \\ Departments of ${ }^{1}$ Cardiology and ${ }^{2}$ Echocardiography Lab, Fujian Medical University Union Hospital, \\ Fujian Institute of Coronary Heart Disease; Departments of ${ }^{3}$ Ultrasonography and ${ }^{4}$ Imaging, \\ Fujian Medical University Union Hospital, Fuzhou, Fujian 350001, P.R. China
}

Received November 1, 2016; Accepted June 15, 2017

DOI: $10.3892 / \mathrm{mmr} .2017 .7314$

\begin{abstract}
The aim of the present study was to investigate the feasibility of using ultrasonography (US) and magnetic resonance (MR) for bimodal molecular imaging of atherosclerotic neovasculature with liquid perfluorocarbon magnetic nanocapsules (NCs) targeted to vascular endothelial growth factor receptor 2 (VEGFR-2). By incorporating perfluorooctyl bromide (PFOB) and superparamagnetic iron oxide (SPIO) into polylactic acid, a SPIO-embedded PFOB NC was constructed; subsequently, a VEGFR-2-targeted NC (VTNC) containing dual detectable probes was created by covalently linking a VEGFR-2 antibody onto the surface of the SPIO-embedded PFOB NC. Target specificity was verified in vitro by incubating VTNC with VEGFR-2 ${ }^{+}$or VEGFR-2- endothelial cells. Rats with vulnerable plaques were assigned to receive either an injection of VTNC (Targeted group; $n=8$ ) or an injection of NC (Nontargeted group; $n=8$ ); control rats also received an injection of VTNC (Control group; $n=8$ ). US and MR imaging of the abdominal aorta were performed to detect VTNC by measuring of the ultrasonic grayscale intensity (GSI) and MR contrast-to-noise ratio (CNR) prior to and at successive time points following VTNC and NC injections. The percent positive
\end{abstract}

Correspondence to: Dr Lianglong Chen, Department of Cardiology, Fujian Medical University Union Hospital, Fujian Institute of Coronary Heart Disease, 29 Xin-Quan Road, Fuzhou, Fujian 350001, P.R. China

E-mail: lianglongchenxh@126.com

Abbreviations: US, ultrasonography; MR, magnetic resonance; VEGFR-2, vascular endothelial growth factor receptor 2; PFOB, perfluorooctyl bromide; SPIO, superparamagnetic iron oxide; VTNC, VEGFR-2-targeted nanocapsule

Key words: ultrasound, magnetic resonance, molecular imaging, atherosclerosis, angiogenesis, vascular endothelial growth factor receptor 2 (VEGFR-2), VEGFR-2-targeted nanocapsules area (PPA) of CD31+ $\left(\mathrm{PPA}_{\mathrm{CD} 31+}\right)$ or VEGFR-2 ${ }^{+}\left(\mathrm{PPA}_{\mathrm{VEGFR}-2+}\right)$ expression was quantified by immunohistochemical staining. CD31 was used to verify the existence of endothelial cells as it is widely expressed on the surface of endothelial cells whether activated or not. The results demonstrated that VTNC was able to highly and selectively detect VEGFR-2 ${ }^{+}$endothelial cells, and GSI, CNR, PPA $\mathrm{CD} 31+$ and PPA $\mathrm{VEGFR}-2+$ were significantly increased in the targeted group compared with the nontargeted and control groups. In the control group, no atherosclerotic plaques or angiogenesis was identified, thus no expression of PPACD31+ and PPAVEGFR-2 (data not shown). There were strong correlations among GSI, CNR, PPA ${ }_{\mathrm{CD} 31+}$ and $\mathrm{PPA}_{\mathrm{VEGFR}-2+}$. In conclusion, two-probe VTNC is feasible for bimodal US and MR molecular imaging of atherosclerotic neovasculature, which may offer complementary information for the more reliable prediction of plaque vulnerability.

\section{Introduction}

Pathophysiological mechanisms underlying atherosclerosis and its thrombotic complications are extremely complex. Luminal thrombosis-induced acute ischemic events are often triggered by the sudden rupture of a vulnerable plaque, which is characterized by having a large lipid core, a thin fibrous cap, high plaque burden and the presence of inflammatory cells (1). Other key high-risk features include: Neovascularization, inflammation and intraplaque hemorrhage (2).

Among the complex mechanisms that may cause plaque rapture, the upregulation of intraplaque angiogenesis and the proliferation of adventitial vasa vasorum have attracted much interest. Intraplaque angiogenesis is closely related to the inflammatory process and may result in erosion of the extracellular matrix, which may subsequently be replaced with fragile neovasculature, resulting in weakened arterial walls or plaque rupture (3). Proliferation of the adventitial vasa vasorum is linked with early plaque formation and extensive neoangiogenesis has previously been associated with vulnerable plaque features $(4,5)$. The mechanisms by which the vasa vasorum may contribute to plaque instability include their roles in leukocyte 
recruitment and intraplaque hemorrhage (3). Intraplaque neoangiogenesis were demonstrated to originate from the adventitial vasa vasorum network and have been associated with inflammatory infiltrates subjacent to advanced plaques $(5,6)$. Owing to their immature and fragile nature, neomicrovessels may locally promote the extravasation of plasma proteins and erythrocytes, an important source of free cholesterol with consequent macrophage infiltration; intraplaque extravasations may be involved in the expansion of the necrotic core, which may subsequently cause abrupt lesion progression (7). Therefore, the direct visualization of neomicrovessels and vasa vasorum may be important for detecting of plaque vulnerability, and the identification of reliable biomarkers that are targeted to plaque neoangiogenesis and the selection of proper imaging modalities are of great importance.

There are numerous candidate biomarkers for molecular imaging of neoangiogenesis, including selectins, integrins, vascular cell adhesion molecule (VCAM) and vascular endothelial growth factor (VEGF). VEGF and VEGF receptors (VEGFRs) serve unique roles in the mediation and promotion of intraplaque neoangiogenesis (8). VEGF is a $45 \mathrm{kDa}$ glycoprotein that is secreted in the vascular wall by endothelial and smooth muscle cells and is a main mediator of angiogenesis and vascular permeability (9). A previous study has confirmed that endothelial cells of novel intraplaque microvessels express higher concentrations of VEGF compared with those in the main arterial lumen, which in turn promotes angiogenesis (10). In addition, VEGFR-2 (a tyrosine kinase receptor), has been reported to be highly expressed on the activated endothelial cell layer, particularly in neonatal blood vessels, which covers the atherosclerotic plaque; whereas the healthy vascular wall expresses low levels of VEGFR-2 on the luminal endothelial cells (11). VEGFR-2 has been revealed to be a key component of the signaling system that regulates proliferation and migration of endothelial and vascular smooth muscle cells, and promotes neovascularization (12). Therefore, VEGFR-2 may be a promising candidate for molecular imaging of intraplaque neoangiogenesis.

Previous studies have focused on VEGFR-2 as a target in preclinical trials for tumor imaging and block tumor angiogenesis, which led to significantly suppressed tumor growth $(13,14)$. However, studies on the imaging of intraplaque neovasculature with VEGFR-2-targeted agents are limited (15). Additionally, despite there being several noninvasive imaging modalities available for the molecular imaging of intraplaque neoangiogenesis, such as magnetic resonance (MR), ultrasonography (US), nuclear techniques, computed tomography angiography (16-18), most studies focused on the use of only one imaging modality, thereby failing to provide complementary insights into neoangiogenesis.

Recent advances in the fabrication of nanomaterials has led to their wide clinical application in diagnostics (19). Perfluorooctyl bromide (PFOB) with its exceptional properties including low surface tension, inertness and biocompatibility, has been encapsulated in biocompatible and biodegradable polymeric shells to form ultrasound contrast agents with significant echogenicity (20). Superparamagnetic iron oxide (SPIO), which is a T2-weighted MRI contrast agent, can offer an intensive negative contrast enhancement of the target lesion in MRI due to its biocompatibility and high sensitivity (21).
The present study hypothesized that intensive intraplaque neoangiogenesis is a biomarker for impending plaque rupture, and VEGFR-2 may be a promising candidate that reflects neoangiogenesis closer to vascular pathological conditions. Intraplaque angiogenesis is associated with plaque rupture, and VEGFR-2 has been confirmed as highly expressed on the activated endothelial cells within plaque (11). The levels of VEGFR-2 may therefore reflect neoangiogenesis in plaque. Therefore, the present study aimed to: i) Examine the reliability of the VEGFR-2-targeted nanocapsule (VTNC) with dual probes of SPIO and PFOB as a contrast agent for molecular imaging of intraplaque neovasculature; and ii) to explore the feasibility and superiority of combined US and MR for in vivo bimodal molecular imaging of atherosclerotic neovasculature by using VTNC.

\section{Materials and methods}

Materials and reagents. 1-(3-Dimethylaminopropyl)-3-ethyl carbodiimide hydrochloride (EDC), N-hydroxysuccinimide (NHS) perfluorooctyl bromide (PFOB) and ethyldiisopropylamine (DIPEA) were purchased from J \& K Scientific Ltd. (Beijing, China). Poly-lactic-co-glycolic acid (PLGA-COOH; 50:50; molecular weight 15,000) was purchased from Daigang Biomaterial Co., Ltd. (Jinan, China); polylactic acid (80k MW) was purchase from Shandong Institute of Medical Instruments (Shandong, China); polyvinyl alcohol (PVA; 86-89\% hydrolyzed; low molecular weight; Alfa Aesar; Thermo Fisher Scientific, Inc., Waltham, MA, USA). Rabbit polyclonal VEGFR-2 primary antibody (ab39256) was from Abcam (Cambridge, MA, USA); secondary fluorescein isothiocyanate (FITC)-labeled goat anti-rabbit immunoglobulin (IgG; zf-0511) was from OriGene Technologies, Inc. (Beijing, China); monoclonal CD31 primary antibody (MAB-0031) was from Maixin Biotech. Co., Ltd (Fuzhou, China); 2-(N-morpholino) ethanesulfonic acid hydrate (MES) was from Best-reagent Co. (Chengdu, China); primary bovine aortic endothelial cells (BAECs) were from Health Science Research Resources Bank (Osaka, Japan); Dulbecco's modified Eagle's medium (DMEM) and fetal bovine serum (FBS) were purchased from Gibco (Thermo Fisher Scientific, Inc.) and deionized (DI) water (18.2 $\mathrm{M} \Omega-\mathrm{cm}$ ) was obtained from Milli-Q Gradient System (Merck KGaA, Darmstadt, Germany).

Iron oxide nanoparticle preparation. Oleic acid-stabilized superparamagnetic iron oxide nanoparticles (SPIOs) were generated by a following a previously described co-precipitation method (22). Briefly, $\mathrm{FeCl}_{3}-6 \mathrm{H}_{2} \mathrm{O}(0.5 \mathrm{M})$ and $\mathrm{FeSO}_{4}-7 \mathrm{H}_{2} \mathrm{O}$ $(0.5 \mathrm{M})$ were dissolved in DI water $(100 \mathrm{ml})$ in a 4-necked flask, followed by the addition of $\mathrm{NH}_{3}-\mathrm{H}_{2} \mathrm{O}$ solution $(30 \mathrm{ml})$ at room temperature under $\mathrm{N}_{2}$ atmosphere. The aqueous solution was heated to $80^{\circ} \mathrm{C}$ for $30 \mathrm{~min}$, and oleic acid $(0.891-0.896 \mathrm{~g} / \mathrm{ml})$ was added with vigorous stirring for an additional $30 \mathrm{~min}$. The precipitate was isolated with a neodymium magnet, washed 3 times with DI water and 3 times with acetone, and dispersed in hexane for subsequent use.

SPIO-embedded PFOB nanocapsule (NC) fabrication. Polymer PLA (100 mg), PLGA-COOH (1.1 mg) and PFOB $(0.1 \mathrm{ml})$ were dissolved in methylene chloride $(3.5 \mathrm{ml})$, and 
combined with oleic acid-stabilized SPIO in hexane $(20 \mathrm{mg}$ $\left.\mathrm{ml}^{-1} ; 0.5 \mathrm{ml}\right)$ and well mixed. SPIO-embedded PFOB NCs were prepared by an adapted oil-in-water $(\mathrm{O} / \mathrm{W})$ emulsion solvent evaporation process as previously described (20). Briefly, the $\mathrm{O} / \mathrm{W}$ emulsion was generated by adding the oil phase from the oleic acid stabilized SPIO-embedded PFOB NCs to an aqueous solution of PVA (2\% w/v; $20 \mathrm{ml})$, followed by continuous probe sonication for $120 \mathrm{sec}$ with a $1.27 \mathrm{~cm}$ diameter titanium alloy horn (Sonicator S-4000; Misonix, Inc.; Qsonica, Newton, CT, USA) using an output amplitude setting of $80 \%$. Subsequently, the emulsion was magnetically stirred at room temperature for $2 \mathrm{~h}$ to evaporate most of the methylene chloride, and the NCs were collected by centrifugation (Avanti J-25 high-speed centrifuge; Beckman Coulter, Inc. Brea, CA, USA) at $21,000 \times \mathrm{g}$ at $15^{\circ} \mathrm{C}$ for $5 \mathrm{~min}$, washed and dispersed with DI water and stored at $4^{\circ} \mathrm{C}$ until use.

VTNC fabrication. Anti-VEGFR-2 antibodies were covalently attached onto the NC surfaces to create VTNC using a previously described carbodiimide technique (23). Briefly, the NCs were washed with MES buffer ( $\mathrm{pH}$ 6.0), collected by centrifugation (96 x $\mathrm{g}$ at room temperature for $3 \mathrm{~min}$ ), activated with a mixture of EDC and NHS (the molar ratio of PLGA-COOH to EDC to NHS was 1:10:20) in MES buffer ( $\mathrm{pH}$ 6.0) for $30 \mathrm{~min}$ at room temperature. The NCs were washed twice with MES ( $\mathrm{pH}$ 6.0) followed by one wash with MES ( $\mathrm{pH}$ 8.0), centrifuged $96 \mathrm{x} \mathrm{g}$ at room temperature for $3 \mathrm{~min}$ to eliminate the remaining unreacted EDC/NHS mixture, and dispersed in MES buffer (pH 8.0). Subsequently, an excess of anti-VEGFR-2 antibodies (molar ratio of PLGA-COOH to anti-VEGFR-2 was 50:1) were immersed into the mixture to ensure that the antibodies fully bound to the PLGA-COOH located on the surface of the nanocapsules. The reaction was incubated for $2 \mathrm{~h}$ at room temperature and stopped by washing twice with phosphate buffered saline (PBS; pH 7.0). The immuno-NCs were collected via centrifugation (96 $\mathrm{x} g$ at room temperature for $3 \mathrm{~min}$ ) and redispersed in $100 \mu \mathrm{l}$ PBS for use (24).

$N C$ characterization and cell culture. The size distribution of NCs was measured by dynamic light scattering using a Nicomp 380 ZLS particle size distribution analyzer (Particle Sizing Systems, Inc., Port Richey, FL, USA). NC morphology was observed and measured with a JEM-100CXII transmission electron microscope (TEM; JEOL, Ltd., Tokyo, Japan). To test if the antibodies were able to covalently attach to the $\mathrm{NCs}$, primary bovine aortic endothelial cells (BAECs) were used.

BAEC cells were cultured in Dulbecco's modified Eagle's medium supplemented with $10 \%$ fetal bovine serum at $37^{\circ} \mathrm{C}$ in $5 \% \mathrm{CO}_{2}$. BAECs $\left(1 \times 10^{6}\right)$ were treated for $24 \mathrm{~h}$ at $37^{\circ} \mathrm{C}$ with or without glucose $(33 \mathrm{mmol} / \mathrm{l})$ to induce VEGFR-2 expression, as previously described (25). Following the induction of VEGFR-2 expression, cells were incubated with VTNC (30 mg/ml) at $4^{\circ} \mathrm{C}$ in $300 \mu \mathrm{l}$ PBS for $1 \mathrm{~h}$. The cells were centrifuged at $96 \mathrm{x} \mathrm{g}$ at room temperature for $3 \mathrm{~min}$ and washed several times with PBS to remove unbound NCs. Cells were incubated with FITC-conjugated goat anti-rabbit IgG antibodies in the dark for $2 \mathrm{~h}$ at room temperature to determine whether the surfaces of fluorescent-labeled immuno-NCs appeared green under fluorescent microscope.
Atherosclerotic animal model. Male Sprague Dawley rats (weight, 246 $\pm 12 \mathrm{~g}, \mathrm{n}=28$ ) were obtained from The Experimental Animal Center of Fujian Medical University (Fuzhou, China) and kept in a pathogen-free facility with a constant humidity $(45 \%)$ and temperature $\left(26^{\circ} \mathrm{C}\right)$ with a 12 -h light/dark cycle and with free access to food and water. A rat model with vulnerable plaque was established as previously described (26). Briefly, following one week of feeding on standard rat chow, all rats were anesthetized with pentobarbital sodium $(0.5 \mathrm{ml} / 100 \mathrm{~g} ; 1 \% \mathrm{w} / \mathrm{v}$ in $0.9 \%$ saline) and underwent abdominal aorta balloon-injury (ABI) $(n=20)$ or sham operation $(n=8)$. The left common carotid artery was dissected and ligated, and an over-the-wire balloon catheter with a balloon of $1.5 \mathrm{~mm}$ was advanced through the left common carotid artery until the abdominal aorta $(\sim 10 \mathrm{~cm})$. The balloon was inflated to a pressure of 12 atm (1.216 MPa) followed by pulling back $5 \mathrm{~cm}$ (corresponding to the target segment from the celiac brunch to iliac artery); this step was repeated five times for ABI. The sham operation was performed by dissection and ligation of the left common carotid artery without ABI. The ABI rats were fed 3\% cholesterol chow for 120 days to create the atherosclerotic model, and the Sham control rats were fed standard chow for 120 days.

Experimental protocol. The study protocol was approved by The Animal Studies Committee, Fujian Medical University Union Hospital (Fuzhou, China). All animal experiments were performed in accordance with the relevant laws and institutional guidelines. The cholesterol-fed rats with ABI (atherosclerotic rats) were randomly placed into 2 groups ( $n=8 /$ group): i) The atherosclerotic/targeted group, which received an intravenous injection of VTNC (30 mg ml$\left.l^{-1} ; 200 \mu \mathrm{l}\right)$; and ii) the atherosclerotic/nontargeted group, which received an intravenous injection of nontargeting NC (30 mg ml $\left.\mathrm{ml}^{-1} ; 200 \mu \mathrm{l}\right)$. In addition, the Sham control rats $(n=8)$ received an injection of VTNC (30 mg ml-1; $200 \mu \mathrm{l}$; control/targeted group).

In vitro imaging of $V T N C$. For US imaging, NCs were prepared in PBS at different concentrations $\left(0,1 \times 10^{4}, 1 \times 10^{5}, 1 \times 10^{6}, 1 \times 10^{7}\right.$ and $1 \times 10^{8}$ particles $/ \mathrm{ml}$ ), injected into a latex tube and imaged using an Aplio 500 Sonographic System (Toshiba Corporation, Tokyo, Japan) with a 12L-5 Linear-Array Transducer (12L-5, Toshiba Corporation). Both pulse inversed harmonic imaging (PIHI) with a mechanical index (MI) of 0.72 and B-mode imaging were performed to obtain the transverse sectional images of the tube. The video intensity was analyzed by the program provided by the US system.

For MRI, the NCs were prepared in PBS at different concentrations $(0,5,10,20,40$ and $80 \mu \mathrm{M})$, scanned with T2-weighted (T2W) imaging with a 3.0 Tesla (T) GE Discovery MR750 MRI scanner (GE Healthcare, Chicago, IL, USA) with a small animal coil using the following settings: Repetition time $(\mathrm{TR})=5,000 \mathrm{msec}$; echo time (TE) between 10.6 and $53 \mathrm{msec}$; field of view $(\mathrm{FOV})=25 \times 62 \mathrm{~mm}$; and slice thickness $(\mathrm{ST})=1.0 \mathrm{~mm}$. All images were stored for off-line analysis for calculating the T2 relaxivity using the analytical software provided with the MRI instrument.

In vivo imaging of VTNC. At the end of day 120, rats were anesthetized with pentobarbital sodium $(0.5 \mathrm{ml} / 100 \mathrm{~g} ; 1 \% \mathrm{w} / \mathrm{v}$ in $0.9 \%$ saline) through intraperitoneal administration. 

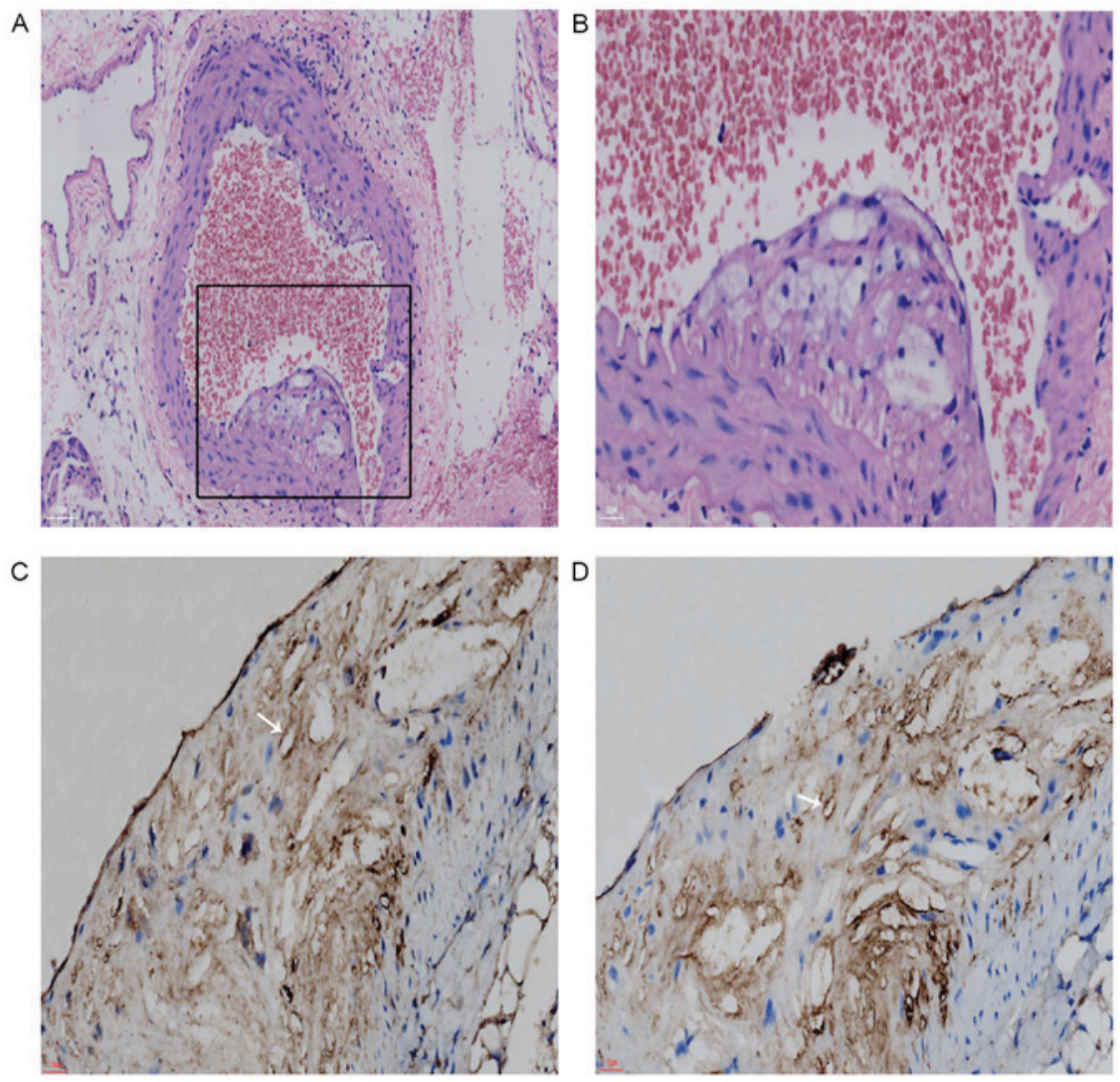

Figure 1. Confirmation of atherosclerotic plaque and neoangiogenesis. (A) Hematoxylin and eosin staining detected an atherosclerotic plaque in transverse abdominal aorta section. Scale bar, $70 \mu \mathrm{m}$. (B) Magnification of the atherosclerotic plaque in (A; box) distinctly exhibiting a typical vulnerable plaque with a thin cap, lipid-rich necrotic core and rich foam cells. (C and D) Immunohistochemical staining demonstrated abundant intraplaque expression of (C) vascular endothelial growth factor receptor 2 (arrow) and (D) CD31 along the luminal endothelial surface (arrow). Scale bar, $20 \mu \mathrm{m}$ in B, C and D.

For US imaging, the rat abdominal aorta below the celiac branch to the iliac artery was interrogated using a Vivid E7 Ultrasound System with a ML6-15 US probe (GE Vingmed Ultrasound AS, Horten, Norway). Tissue harmonic imaging (THI) modality was used with the following consistent settings: $\mathrm{MI}=1.6$; frequency=15 MHz; gain=52; and depth=2 $\mathrm{cm}$. A set of images were obtained prior to injection and at 2.5, 5, 15, 30 and $60 \mathrm{~min}$, and at 2, 4 and $24 \mathrm{~h}$ post-NC injection of NCs suspension $\left(30 \mathrm{mg} \mathrm{ml}^{-1}, 200 \mu \mathrm{l}\right)$. The mean grayscale intensity (GSI) in the region of interest (ROI) was measured prior to and following contrast enhancement by using the histogram tool in Photoshop version 7.01 (Adobe Systems, Inc., Beijing, China), and the change in GSI at each time point was calculated to assess the relative retention of NCs in the plaques.

For MRI, the rat abdominal aorta below the celiac branch to the iliac artery was scanned using a 3.0T GE Discovery MR750 MRI scanner with a knee coil. T2W scanning was used under the following 3D time-of-flight sequence parameters: $\mathrm{TR} / \mathrm{TE}=22 / 5.7 \mathrm{msec}$; flip=15; FOV=8x6 cm; matrix $=512 \times 512$; and $\mathrm{ST}=1.2 \mathrm{~mm}$. A set of images was recorded prior to injection and at 1, 4 and $24 \mathrm{~h}$ post-injection of NCs. Following acquisition of the T2W images, recognition of the aortic wall and signal intensity in ROI were performed with the analysis programs provided by the MRI scanner. The threshold of NCs was defined to be 3 standard deviations below the mean signal intensity of the pre-contrast abdominal aorta on signal intensity histograms. The threshold was applied to sequential abdominal aorta MRI slices to determine the mean area of NC low-signal contrast. The contrast-to-noise ratio (CNR) of each ROI was compared prior to and following contrast. CNR was calculated as previously described (27): $\mathrm{CNR}=($ blood pool signal-lesion signal)/standard deviation of the noise.

Histological analysis. Following US and MR imaging, rats were sacrificed, abdominal aorta were removed, cut into $2.5 \mathrm{~mm}$ serial sections, fixed overnight in $4 \%$ paraformaldehyde at $4^{\circ} \mathrm{C}$, with a second overnight tissue processing and dehydration, and embedded in paraffin. The slides were stained with hematoxylin and eosin. The luminal surface was observed by microscopy (Olympus Corporation, Tokyo, Japan).

Immunohistochemistry. Immunohistochemical staining for VEGFR-2 and CD31 were performed as follows. Following fixation with $4 \%$ paraformaldehyde at $4^{\circ} \mathrm{C}$ for $12 \mathrm{~h}$, abdominal aorta samples were processed for paraffin-embedded slides. The slides were subjected to antigen retrieval, and the endogenous peroxidase activity was quenched with hydrogen peroxide. After blocking nonspecific proteins with normal serum in PBS (0.1\% Tween 20$)$, the slides were incubated with rabbit polyclonal antibodies against VEGFR-2 and CD31 (all at 1:300 dilution). Following washing with PBS, the slides were incubated with biotinylated secondary antibody followed by conjugated horseradish peroxidase (HRP)-labelled 

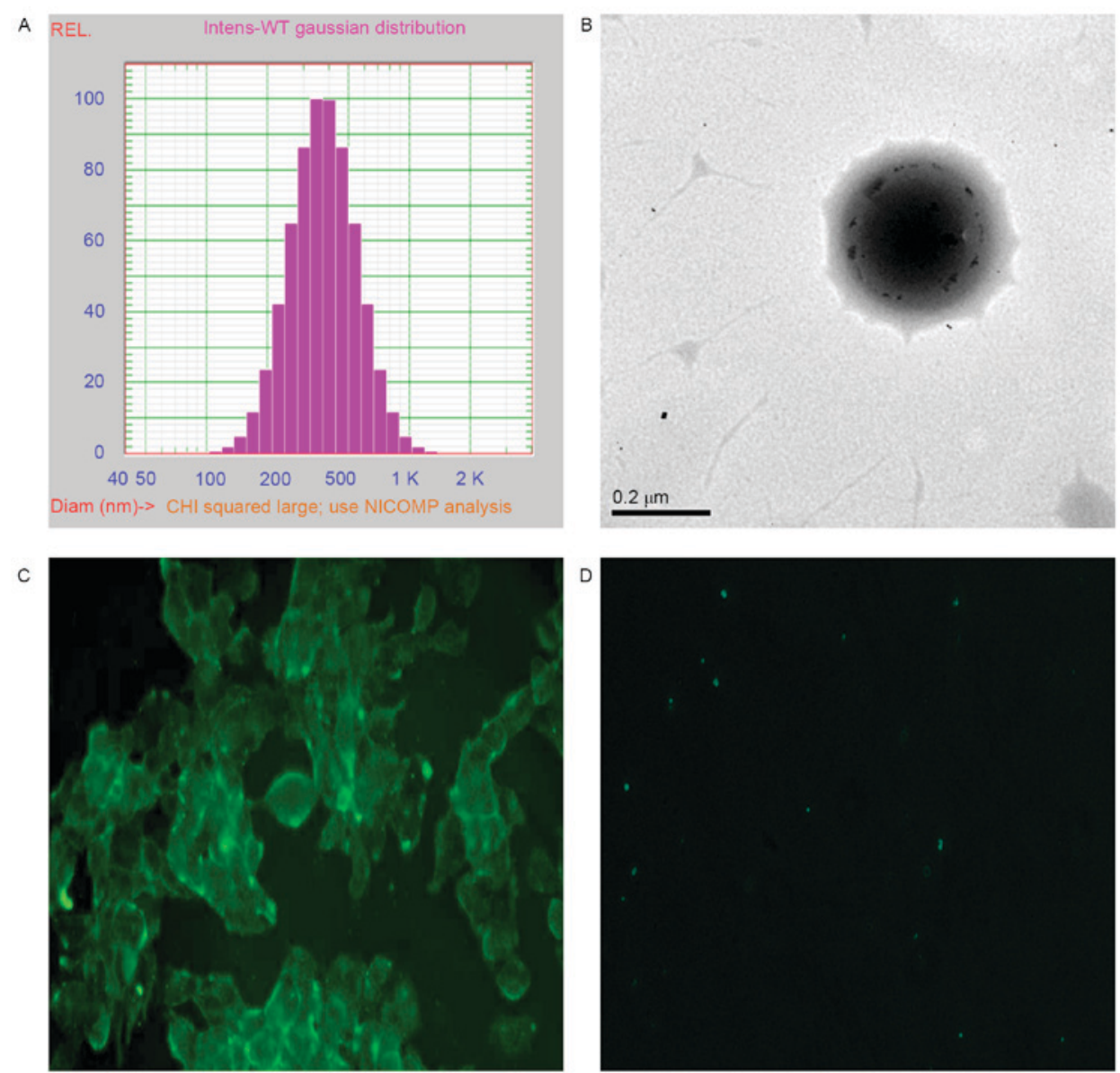

Figure 2. Characterization of the VEGFR-2-targeted NCs. (A) Monodispersion of NCs with mean diameter of $400 \mathrm{~nm}$. (B) Encapsulation of SPIO in the NCs detected by TEM (dark grey spots). (C and D) Immunodetection of VEGFR-2 expression in primary bovine aortic endothelial cells treated either (C) with high glucose (strong fluorescence, indicating high VEGFR-2 expression) or (D) without high glucose (weak or no fluorescence, indicating low VEGFR-2 expression). NC, nanocapsule; VEGFR-2, vascular endothelial growth factor receptor 2.

streptavidin (Dako; P039701-2; 1:400; Agilent Technologies, Inc., Santa Clara, CA, USA) at $37^{\circ} \mathrm{C}$ for $60 \mathrm{~min}$ and then washed with PBS. The slides were then incubated with diamino-benzidine (Sigma-Aldrich; Merck KGaA) as the chromogen at $37^{\circ} \mathrm{C}$ for $10 \mathrm{~min}$, followed by counterstaining with diluted Harris hematoxylin (Sigma-Aldrich; Merck $\mathrm{KGaA}$ ). Images of the stained tissue sections were captured with an optical microscope (Olympus Corporation, Tokyo, Japan) for computerized image analysis with Image-Pro Plus version 6.0 (Media Cybernetics, Inc., Rockville, MD, USA). The brown-stained areas in the tissue sections were defined as $\mathrm{CD} 31^{+}$or VEGFR-2 $2^{+}$, and were easily identified from the other tissues. The percent positive area (PPA) of $\mathrm{CD} 31^{+}$or VEGFR-2 $2^{+}$expression $\left(\mathrm{PPA}_{\mathrm{CD} 31+}\right.$ or $\mathrm{PPA}_{\mathrm{VEGFR}-2+}$; respectively) was calculated by [(positive area/total area)x100\%] and adopted as a parameter for reflection of neoangiogenesis as previously described (28).

Control of analytic quality. Consideration of the comparability and consistency required for parametric analysis, a similar ROI was used for US/MR imaging, and histopathological sections were selected at the same anatomical sites with the most severe plaque formation. All data were analyzed by two observers blinded to each other's results and the results among imaging or histological measurements.
Statistical analysis. All analysis was performed with SPSS 19.0 software package (IBM Corp., Armonk, NY, USA). Continuous data were expressed as the mean \pm standard deviation. One-way of analysis of variance was used to analyze differences among groups, followed by Fisher's least significant difference test between groups if significant. Correlations between imaging and histological parameters were assessed by Pearson's correlation analysis. $\mathrm{P}<0.05$ was considered to indicate a statistically significant difference.

\section{Results}

Confirmation of vulnerable plaques. Of the 20 rats that underwent ABI surgery, 1 failed to recover from anesthesia and 3 perished during the operation. The remaining 16 rats were fed $3 \%$ cholesterol chow for 120 days to develop aortic plaques, most of which were vulnerable plaques with a thin cap and large lipid core filled with foam cells, confirmed pathologically and immunohistochemically (Fig. 1A-D). Sham rats fed standard chow did not develop notable plaques (data not shown).

Characterization of VTNC. Measurement of NC particle size distribution indicated a monodispersion with an average diameter of $404.4 \pm 153.7 \mathrm{~nm}$ (Fig. 2A). TEM imaging of NCs demonstrated dark grey spots within the NCs, which 
Table I. Comparison of the average and peak GSI and CNR among the three groups.

\begin{tabular}{|c|c|c|c|}
\hline Measurement & Atherosclerotic/targeted & Atherosclerotic/nontargeted & Control/targeted \\
\hline \multicolumn{4}{|l|}{ GSI (dB) } \\
\hline Baseline & $39.37 \pm 2.14$ & $41.25 \pm 2.82$ & $38.29 \pm 2.89$ \\
\hline Post-injection mean & $65.09 \pm 6.21^{\mathrm{a}}$ & $46.66 \pm 4.01^{\mathrm{b}}$ & $46.02 \pm 4.19^{\mathrm{b}}$ \\
\hline Peak & $69.44 \pm 4.45^{\mathrm{a}}$ & $45.24 \pm 1.92^{\mathrm{b}}$ & $44.78 \pm 4.59^{b}$ \\
\hline \multicolumn{4}{|l|}{ CNR } \\
\hline Baseline & $42.06 \pm 6.03$ & $47.53 \pm 4.19$ & $44.58 \pm 3.63$ \\
\hline Post-injection mean & $74.02 \pm 11.46^{\mathrm{a}}$ & $48.86 \pm 4.07^{\mathrm{b}}$ & $46.88 \pm 4.31^{\mathrm{b}}$ \\
\hline Peak & $87.51 \pm 6.63^{\mathrm{a}}$ & $49.22 \pm 3.19^{\mathrm{b}}$ & $47.12 \pm 5.19^{\mathrm{b}}$ \\
\hline
\end{tabular}

Data are expressed as the mean \pm standard deviation; $\mathrm{n}=8$ /group; ${ }^{\mathrm{a}} \mathrm{P}<0.01$ vs. baseline; ${ }^{\mathrm{b}} \mathrm{P}<0.01$ vs. atherosclerotic/targeted group. $\mathrm{CNR}$, contrast-to-noise ratio; GSI, grayscale intensity.
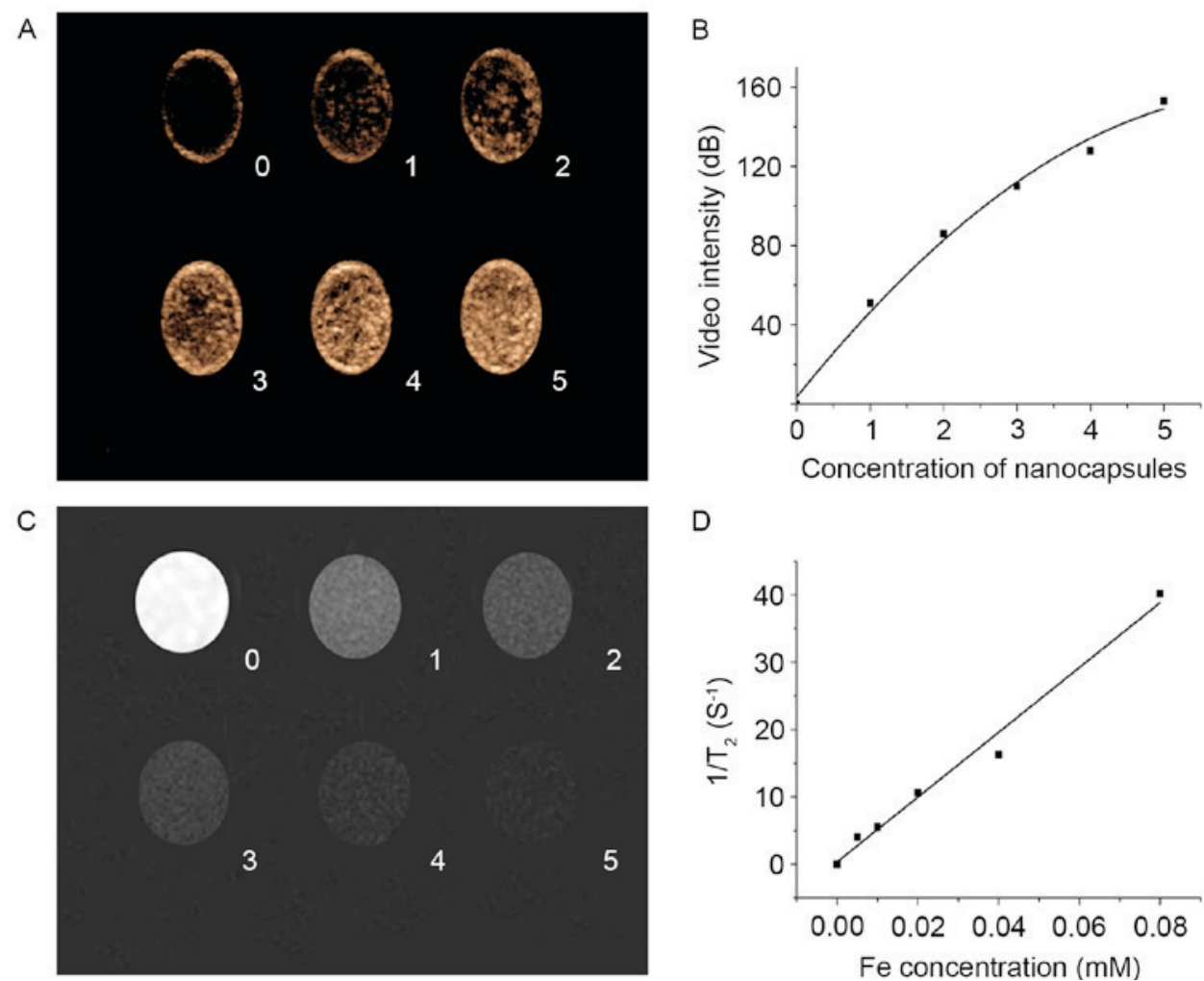

Figure 3. In vitro acoustic or magnetic feature of nanocapsules. (A and B) Notable contrast enhancement in a dose-dependent manner detected with ultrasonographic-pulse inversed harmonic imaging. NCs concentration of sample $0-5: 0,1 \times 10^{4}, 1 \times 10^{5}, 1 \times 10^{6}, 1 \times 10^{7}$ and $1 \times 10^{8}$ particles $\left./ \mathrm{ml}\right)$. (C and $\left.\mathrm{D}\right)$ Prominent contrast attenuation in a dose-dependent manner detected with T2-weighted imaging. Fe concentration of sample 0-5: 0,5, 10, 20,40 and 80 $\mu \mathrm{M}$ ).

indicated the successful encapsulation of SPIO within the NCs (Fig. 2B). Immunodetection with a fluorescent microscope revealed that the targeted VTNCs were able to selectively target VEGFR-2-expression BAECs, in which high or low expression was noted with or without high glucose treatment (Fig. 2C and D, respectively). These results indicated the successful and specific conjunction of anti-VEGFR-2 antibodies onto NCs.

In vitro acoustic or magnetic feature of NCs. US and MR imaging were performed to test the bimodal imaging capability of SPIO-embedded PFOB NCs loaded in the latex tubes. Contrast enhancement in a dose-dependent manner was detected with US-PIHI imaging (Fig. 3A and B); contrast attenuation in a dose-dependent manner was detected with MR-T2W scanning (Fig. 3C and D). In addition to excellent echo-enhancement, high T2 relativity of $421 \mathrm{mM}^{-1} \mathrm{~s}^{-1}$, which is more than double the commercial SPIO-based MR agent Resovist (185.8 $\left.\pm 9.3 \mathrm{Mm}^{-1} \mathrm{~s}^{-1}\right)(29)$, enabled in vitro bimodal detection of SPIO-embedded PFOB NCs with US and MR imaging.

In vivo US imaging of VTNC. US-THI imaging was performed in rats to interrogate contrast enhancement in the targeted 
A
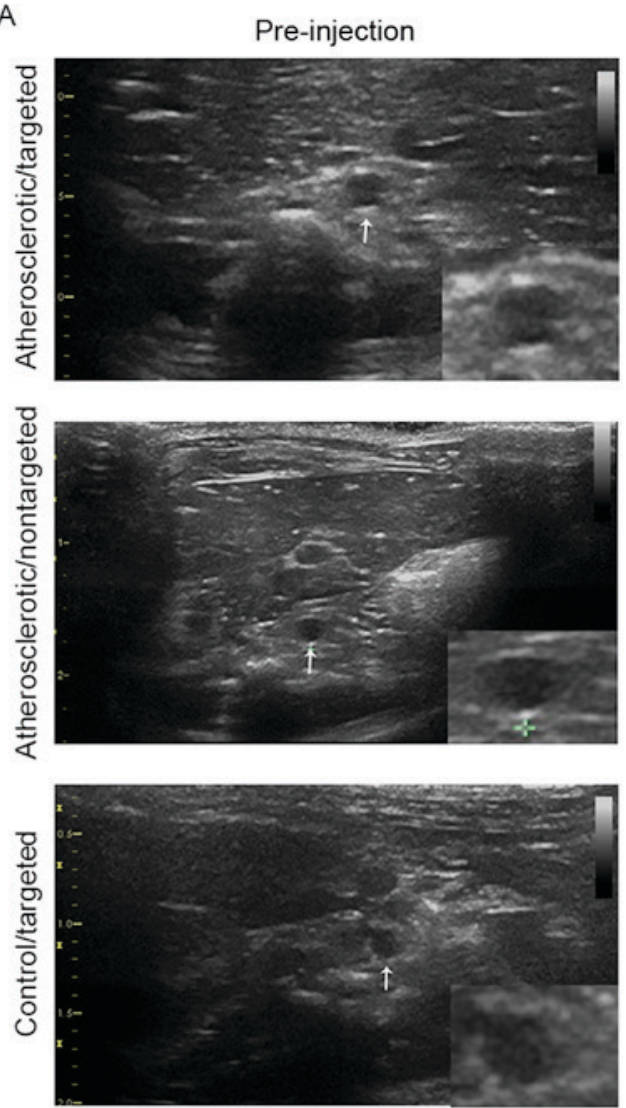

Post-injection
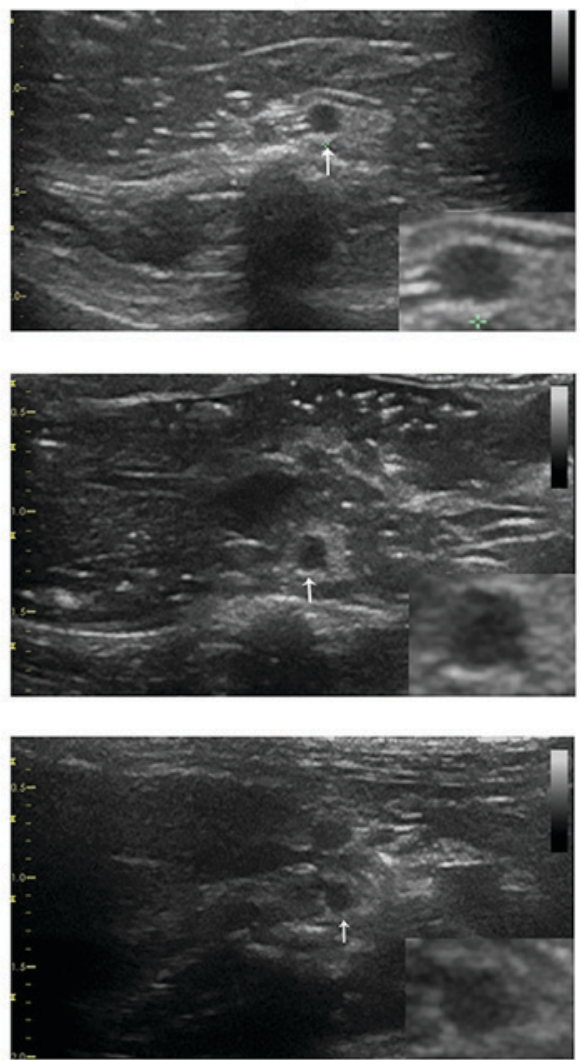

B

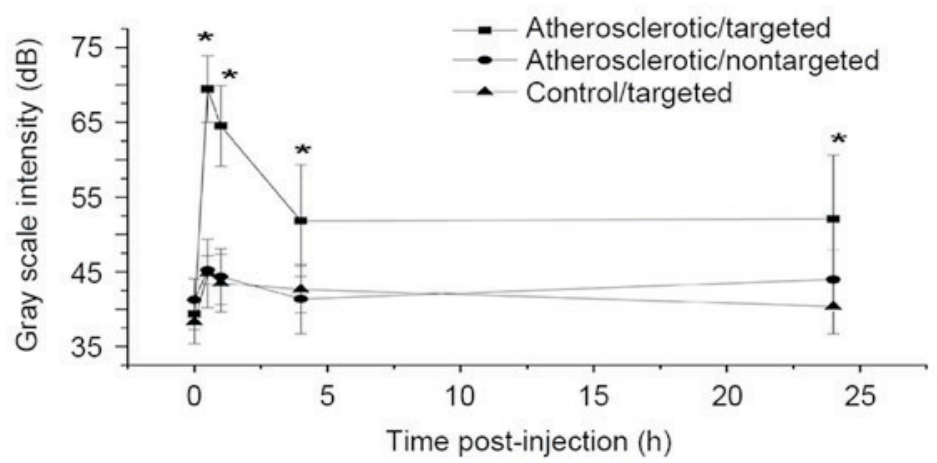

Figure 4. In vivo US-THI of VTNC. (A) Representative transverse section of the abdominal aorta by US-THI imaging in rats from different groups. There was a hyperechoic plaque (arrow) detected in the atherosclerotic/targeted and the atherosclerotic/nontargeted rat; no detectable plaques were observed in the control/targeted rat (left panels). Intraplaque contrast-enhancement was only observed in atherosclerotic/targeted rat following VTNC injection (top panel, right; arrow). No significant plaque enhancement was observed in the other 2 groups following injection (middle and bottom panel, right; arrow). (B) Time-intensity curve demonstrated contrast enhancement in the three groups. "P<0.05 vs. atherosclerotic/nontargeted or control/targeted group. US-THI, ultrasonography-tissue harmonic imaging; VTNC, vascular endothelial growth factor receptor 2-targeted nanocapsule.

segment of rat abdominal aorta prior to and 10, 20, 30, 40, 50, $60 \mathrm{~min}$ and at 2, 4 and $24 \mathrm{~h}$ following the administration of NCs. Hyperechoic plaques were detected in all atherosclerotic rats, although notable enhancement of echoic intensity and massive retention of VTNC in atherosclerotic plaques were observed only in the atherosclerotic/targeted group (Fig. 4A). The baseline GSI was compared among the three groups, administration of NCs significantly increased the mean and peak GSI in the atherosclerotic/targeted group, but not in the atherosclerotic/nontargeted or control/targeted group (Table I). Time-intensity curve analysis clearly indicated that there was higher peak intensity and longer duration of contrast enhancement in the atherosclerotic/targeted group compared with the atherosclerotic/nontargeted and the control/targeted groups (Fig. 4B).

In vivo MRI of VTNC. T2W imaging was used to scan the targeted segment of rat abdominal aorta prior to and 1, 4 and $24 \mathrm{~h}$ following injection of NCs. Aortic plaques were identified in all atherosclerotic rats but not control rats, whereas significant $\mathrm{T} 2 \mathrm{~W}$ signal attenuation was only detected in rats in the atherosclerotic/targeted group (Fig. 5A). Comparing the baseline CNR among the three groups revealed that the injection of NCs significantly attenuated the mean and the 
A
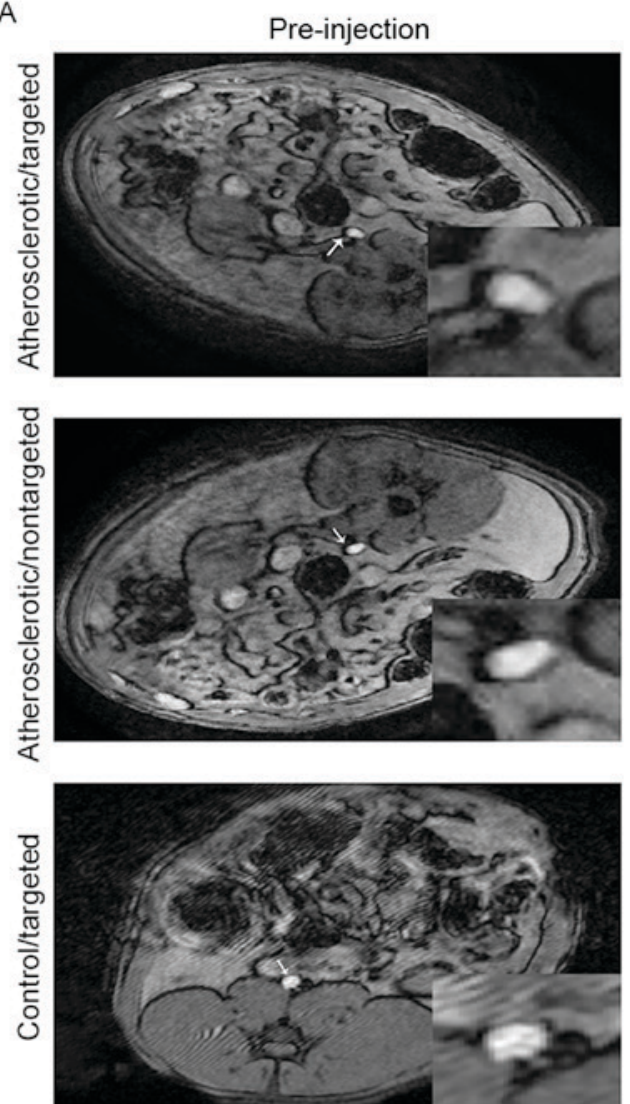
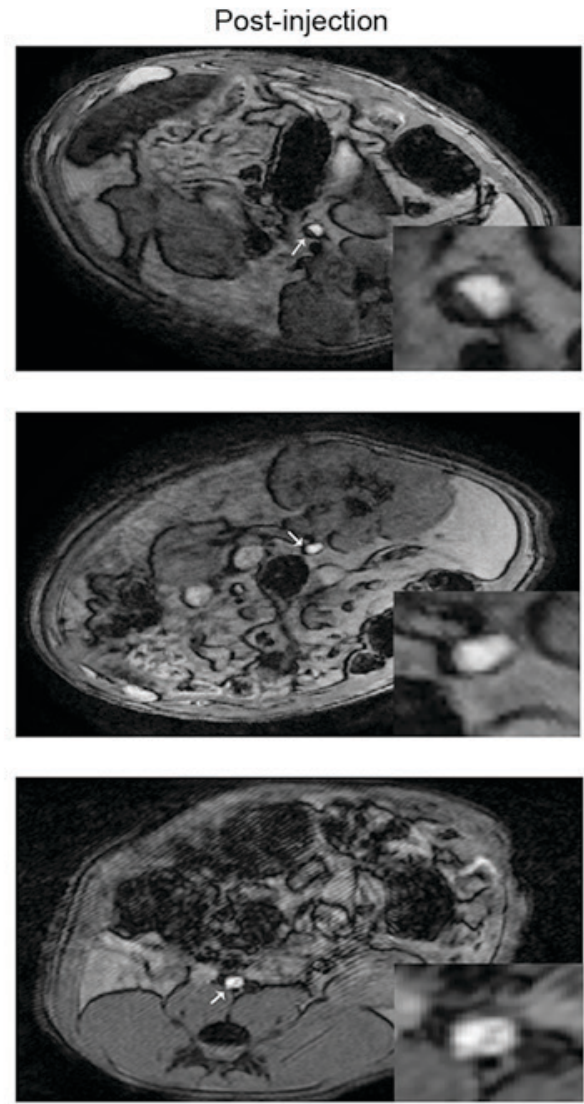

B

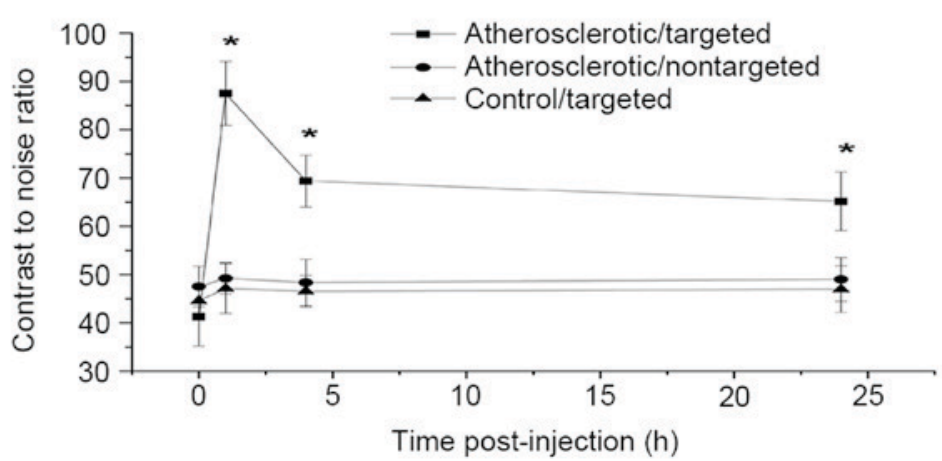

Figure 5. In vivo magnetic resonance imaging of VTNC. (A) Representative transverse scanning of the abdominal aorta by T2-weighted imaging in rats from the three different groups. There was a low-signal plaque (arrow) detected in the atherosclerotic/targeted or atherosclerotic/nontargeted rats which exhibited increased wall thickness and pronounced plaque formation compared with the control/targeted group (left panels); intraplaque magnetic contrast-attenuation was only observed in atherosclerotic/targeted rats following VTNC injection (top panel right; arrow). While in the atherosclerotic/nontargeted and control/targeted group, no significant T2 signal change was observed after injection (middle and bottom panel, right; arrow). (B) Time-intensity curve demonstrates $24 \mathrm{~h}$ contrast attenuation in the groups. ${ }^{~} \mathrm{P}<0.05$ vs. atherosclerotic/nontargeted or control/ targeted group. VTNC, vascular endothelial growth factor receptor 2-targeted nanocapsule.

peak CNR in the atherosclerotic/targeted group, but not in the atherosclerotic/nontargeted or control/targeted group (Table I). Time-attenuation curve analysis indicated a higher peak CNR and longer duration of T2W signal attenuation in the atherosclerotic/targeted group compared with the atherosclerotic/nontargeted or the control/targeted group (Fig. 5B).

Intraplaque VEGFR-2 expression and neovascularization. Immunohistochemical staining indicated that there were notable upregulation of VEGFR-2 expression and proliferation of neonatal vascular endothelial cells within the plaques in the atherosclerotic rats (Fig. 1C and D). Further analysis of the positive expressions revealed that $\mathrm{PPA}_{\mathrm{VEGFR}-2+}$ was $44.5 \pm 2.9 \%$ in the atherosclerotic/targeted group and $43.1 \pm 3.2 \%$ in the atherosclerotic/nontargeted group $(\mathrm{P}>0.05)$. Similarly, $\mathrm{PPA}_{\mathrm{CD} 31+}$ was $46.0 \pm 3.0 \%$ in the atherosclerotic/targeted group and $44.6 \pm 3.5 \%$ in the atherosclerotic/nontargeted group ( $\mathrm{P}>0.05)$. The control/targeted group did not develop atherosclerotic plaques (data not shown).

Correlations between in vivo US/MR imaging and immunostaining parameters. In the atherosclerotic/targeted group, there were strong correlations between peak GSI and PPA VEGFR-2+ $(\mathrm{r}=0.81 ; \mathrm{P}<0.05)$ and between GSI and $\mathrm{PPA}_{\mathrm{CD} 31+}(\mathrm{r}=0.73$; 

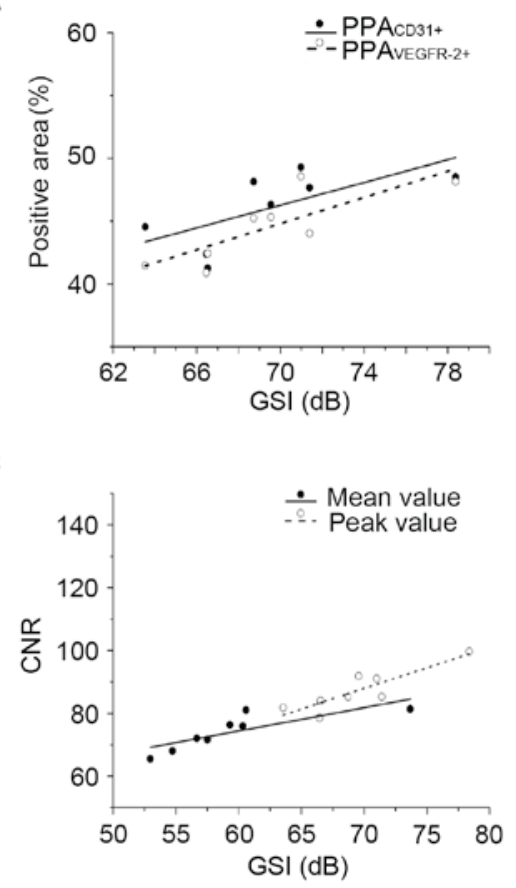

B

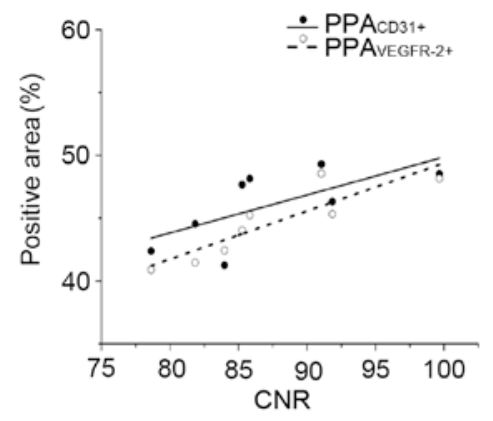

$\mathrm{D}$

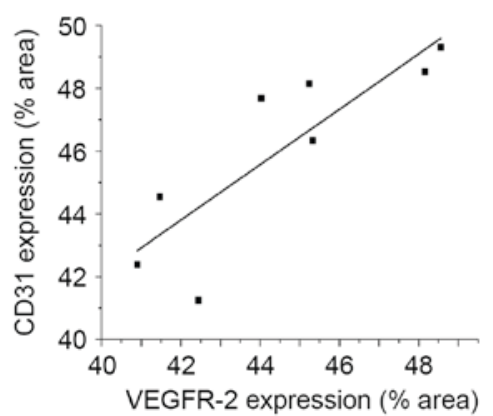

Figure 6. Correlations between in vivo US/MR imaging and immunostaining parameters in the atherosclerotic/targeted group. (A) Linear regression analysis indicated that peak GSI was highly correlated with $\mathrm{PPA}_{\mathrm{VEGFR-2+}}(\mathrm{r}=0.81 ; \mathrm{P}<0.05)$ and $\mathrm{PPA}_{\mathrm{CD} 31+}(\mathrm{r}=0.73 ; \mathrm{P}<0.05)$. (B) Linear regression analysis indicated that peak CNR was highly correlated with $\mathrm{PPA}_{\mathrm{VEGFR}-2+}(\mathrm{r}=0.89 ; \mathrm{P}<0.01)$ and $\mathrm{PPA}_{\mathrm{CD} 31+}(\mathrm{r}=0.72 ; \mathrm{P}<0.05)$. (C) Linear regression analysis of the correlation between peak GSI and CNR $(r=0.87 ; \mathrm{P}<0.01)$ and mean GSI and CNR $(\mathrm{r}=0.82 ; \mathrm{P}<0.05)$. (D) Linear regression analysis of the correlation between $\mathrm{PPA}_{\mathrm{VEGFR}-2+}$ and $\mathrm{PPA}_{\mathrm{CD} 31+}(\mathrm{r}=0.84 \mathrm{P}<0.01)$. US, ultrasonography; MR, magnetic resonance; $\mathrm{CNR}$, contrast-to-noise ratio; GSI, grayscale intensity; VEGFR-2, vascular endothelial growth factor receptor 2 .

$\mathrm{P}<0.05$; Fig. 6A). Correlations were also detected between peak $\mathrm{CNR}$ and $\mathrm{PPA}_{\mathrm{VEGFR}-2+}(\mathrm{r}=0.89 ; \mathrm{P}<0.01)$ and $\mathrm{CNR}$ vs. $\mathrm{PPA}_{\mathrm{CD} 31+}$ $(\mathrm{r}=0.72 ; \mathrm{P}<0.05$; Fig. 6B). Peak GSI exhibited a strong correlation with $\mathrm{CNR}(\mathrm{r}=0.87 ; \mathrm{P}<0.01)$ and the mean GSI with $\mathrm{CNR}$ $\left(\mathrm{r}=0.82\right.$; $\mathrm{P}<0.05$; Fig. 6C). $\mathrm{PPA}_{\mathrm{VEGFR}-2+}$ and $\mathrm{PPA}_{\mathrm{CD} 31+}$ expression also exhibited a strong correlation $(\mathrm{r}=0.84$; $\mathrm{P}<0.01$; Fig. $6 \mathrm{D})$. Conversely, no correlations were detected between the imaging and histological parameters in the atherosclerotic/nontargeted group or the control/targeted group.

\section{Discussion}

Over the past few decades an increasing number of studies from clinical and basic research have improved our knowledge and understanding of the pathophysiological mechanisms that underlie atherosclerosis and the associated thrombotic complications. The focus on stenotic severity of atherosclerotic plaque has been replaced with a new concept that suggested that clinical complications of atherosclerosis, particularly thrombotic complications, are mainly determined by the intramural plaque composition rather than the luminal stenotic severity itself $(1,2)$. The identification of potential molecular targets that may accompany plaque vulnerability and an improvement in the detection capabilities of these molecular targets are gaining more interest (30-32). Although there are several noninvasive molecular imaging modalities available, including US, MR, computed tomography (CT), electrical capacitance tomography and positron emission tomography (PET), determining the optimal molecular targets with suitable detecting techniques remains challenging owing to multifaceted mechanisms that are associated with plaque vulnerability. Nonetheless, intraplaque angiogenesis with proliferation of the adventitial vasa vasorum, one of major mechanisms of plaque vulnerability, is a much easier to target (4).

The present study was the first, to the best of the authors' knowledge, to create VTNC with two probes (PFOB and SPIO) for bimodality US and MR molecular imaging of intraplaque neovasculature. Three main results were obtained from the present study: i) VTNC exhibited a strong capability with highly specific binding efficiency to BAECs expressing VEGFR-2 in vitro; ii) VTNC had a robust ability to target intraplaque neomicrovessels expressing VEGFR-2 with long-term accumulation in vivo; and iii) two-probe VTNC was able to be detected by either US or MR imaging with a broader detectable time-window. Based on the present results, VTNC combined with US and MR imaging provided an optimal combination, which enabled reliable imaging of intraplaque neovasculature and more precise prediction plaque vulnerability.

A number of nontargeted contrast agents have been investigated in previous studies, although they were reported to only enhance the capability for detection of intraplaque neovasculature to a certain but limited extent (33). A major reason for the poor ability to detect neoangiogenesis is that such agents possess only first-pass rather than retention or binding effects, thus affording a very narrow detectable time-window. For these reasons, targeted contrast agents are becoming more commonly used. There are numerous biomarkers, such as P-selectin, $\alpha v \beta 3$ integrin, VCAM-1, ICAM-1 or Profilin-1, that have been used to construct targeted agents for molecularly imaging atherosclerosis (34-37). However, most of 
these biomarkers offered only a single-imaging modality of MR, CT or PET-CT, and fail to provide complementary information into the atherosclerotic process. Although some previous studies have committed to the bimodal imaging of atherosclerosis by combining MR and either nuclear or fluorescence imaging, they were eventually demonstrated to be costly, radioactive and non-real time $(14,37,38)$, and none of them used VEGFR-2 as a candidate to image intraplaque neovasculature with bimodal imaging.

Intraplaque angiogenesis has been demonstrated to be mainly promoted by VEGF, which is overexpressed as atherosclerosis progresses and is primarily mediated by VEGFR-2 (39). VEGFR-2 is widely expressed in neovascular endothelial cells and functions by binding its dominant ligand VEGF, which causes the proliferation of endothelial and vascular smooth muscle cells (40). Accordingly, construction of nanoparticles with detectable probes targeted to VEGFR-2 was theoretically superior and technically promising, and enabled the present study to detect neovascular endothelial cells or, more precisely, to molecularly interrogate intraplaque neovasculature.

As verified in the in vitro experiment, the created VTNCs were monodispersed with a mean diameter of $\sim 400 \mathrm{~nm}$, exhibited strong targeting capability with highly specific binding efficiency to BAECs, and had substantial contrast enhancement under US-PIHI or MR-T2W imaging. Notably, results from the in vivo study revealed that the VTNC exhibited excellent retention ability, as indicated by the strong peak or mean intensity with favorable contrast-enhancing duration in the time-intensity curves. These results suggested that the VTNCs possess not only first-pass effect, but also high binding efficiency and affinity targeted to the neovascular endothelial cells, thus making VTNCs more suitable for molecularly targeted imaging of intraplaque neovasculature.

In addition to having the properties of a targeted biomarker, the detectable probes carried by the VTNCs and the detection tools available are also crucial for precisely detecting intraplaque neoangiogenesis. It is desirable not only to obtain information as accurate as possible in technical view, but also to acquire data as convenient as possible from a practical point. Therefore, the use of at least two imaging modalities would be expected to create an efficient combination to detect their respective probes. Although US exhibits suboptimal spatial resolution, it is the more attractive modality of the noninvasive imaging techniques, owing to its convenient, real-time and low-cost features (41); conversely, MRI possesses excellent spatial resolution, but has low temporal resolution, is costly and examination is time-consuming (42). Integration of US and MR imaging may satisfy the requirements for precisely detecting intraplaque neoangiogenesis by compensating for their individual weaknesses. The proper selection of detectable probes for each imaging modality may present another issue. Among the numerous available probes, PFOB with hyperechoic property and SPIO with superparamagnetic features have been proven to be the most suitable for US or MR detecting, respectively $(21,43)$.

As demonstrated by the in vivo experiments in the present study, both US-derived mean and peak GSI and MR-derived mean and peak CNR were much higher in the atherosclerotic/targeted group compared with the atherosclerotic/nontargeted or control/targeted group. Of all parameters examined, these were highly correlated with intraplaque VEGFR-2 expression and neovascular formation, which suggested that the bimodalities of US and MR imaging may be sensitive and specific for detecting PFOB and SPIO carried by VTNC, respectively. Additionally, the present study demonstrated that there was a broader detectable time-window (at least $24 \mathrm{~h}$ ) for the detection of VTNC using US imaging, which favored continuous and repeated examination and may improve accuracy for imaging intraplaque neovasculature.

The results of the present study may offer a number of benefits; however, there are still some limitations. Although the use of the dual-probe VTNC was feasible for US and MR bimodal imaging of intraplaque neovasculature in the abdominal aorta, it remains difficult to detect coronary plaques due to the constant beating of the heart. In addition, although merging of the images of the same section from the two imaging modalities may improve the accuracy of detection, this remains technically difficult based on the current techniques. This current technique bottleneck may soon be resolved with the introduction of fast high-field MRI scanners and ultrasonographs with high spatial and temporal resolution.

In conclusion, VTNC was associated with robust targeting capability and excellent intraplaque retention ability. The use of VTNCs carrying two probes (PFOB and SPIO) was demonstrated to be technically feasible with a broader detectable time-window for bimodal US and MR molecular imaging of intraplaque neovasculature, which may offer complementary information for a more reliable prediction of plaque vulnerability.

\section{References}

1. Hansson GK, Libby P and Tabas I: Inflammation and plaque vulnerability. J Intern Med 278: 483-493, 2015.

2. Falk E, Nakano M, Bentzon JF, Finn AV and Virmani R: Update on acute coronary syndromes: The pathologists' view. Eur Heart J 34: 719-728, 2013.

3. Haasdijk RA, Den Dekker WK, Cheng C, Tempel D, Szulcek R, Bos FL, Hermkens DM, Chrifi I, Brandt MM, Van Dijk C, et al: THSD1 preserves vascular integrity and protects against intraplaque haemorrhaging in ApoE ${ }^{-/-}$mice. Cardiovasc Res 110: 129-139, 2016.

4. Kolodgie FD, Gold HK, Burke AP, Fowler DR, Kruth HS, Weber DK, Farb A, Guerrero LJ, Hayase M, Kutys R, et al: Intraplaque hemorrhage and progression of coronary atheroma. N Engl J Med 349: 2316-2325, 2003.

5. Moreno PR, Purushothaman KR, Zias E, Sanz J and Fuster V: Neovascularization in human atherosclerosis. Curr Mol Med 6: 457-477, 2006

6. Taruya A, Tanaka A, Nishiguchi T, Matsuo Y, Ozaki Y, Kashiwagi M, Shiono Y, Orii M, Yamano T, Ino Y, et al: Vasa Vasorum Restructuring in Human Atherosclerotic Plaque Vulnerability: A clinical optical coherence tomography study. J Am Coll Cardiol 65: 2469-2477, 2015.

7. Chang SH, Feng D, Nagy JA, Sciuto TE, Dvorak AM and Dvorak HF: Vascular permeability and pathological angiogenesis in caveolin-1-null mice. Am J Pathol 175: 1768-1776, 2009.

8. Michel JB, Martin-Ventura JL, Nicoletti A and Ho-Tin-Noe B: Pathology of human plaque vulnerability: Mechanisms and consequences of intraplaque haemorrhages. Atherosclerosis 234: 311-319, 2014.

9. Brogi E, Wu T, Namiki A and Isner JM: Indirect angiogenic cytokines upregulate VEGF and bFGF gene expression in vascular smooth muscle cells, whereas hypoxia upregulates VEGF expression only. Circulation 90: 649-652, 1994. 
10. Ahmad S, Hewett PW, Wang P, Al-Ani B, Cudmore M, Fujisawa T, Haigh JJ, le Noble F, Wang L, Mukhopadhyay D and Ahmed A: Direct evidence for endothelial vascular endothelial growth factor receptor-1 function in nitric oxide-mediated angiogenesis. Circ Res 99: 715-722, 2006.

11. Belgore F, Blann A, Neil D, Ahmed AS and Lip GY: Localisation of members of the vascular endothelial growth factor (VEGF) family and their receptors in human atherosclerotic arteries. J Clin Pathol 57: 266-272, 2004.

12. Perrot-Applanat M and Di Benedetto M: Autocrine functions of VEGF in breast tumor cells: Adhesion, survival, migration and invasion. Cell Adh Migr 6: 547-553, 2012.

13. Choi HS, Kim MK, Lee K, Lee KM, Choi YK, Shin YC, Cho SG and Ko SG: SH003 represses tumor angiogenesis by blocking VEGF binding to VEGFR2. Oncotarget 7: 32967-32979, 2016.

14. Mancini M, Greco A, Salvatore G, Liuzzi R, Di Maro G Vergara E, Chiappetta G, Pasquinelli R, Brunetti A and Salvatore M: Imaging of thyroid tumor angiogenesis with microbubbles targeted to vascular endothelial growth factor receptor type 2 in mice. BMC Med Imaging 13: 31, 2013.

15. Tekabe Y, Kollaros M, Zerihoun A, Zhang G, Backer MV, Backer JM and Johnson LL: Imaging VEGF receptor expression to identify accelerated atherosclerosis. EJNMMI Res 4: 41, 2014

16. Wang K, Pan D, Schmieder AH, Senpan A, Caruthers SD, Cui G, Allen JS, Zhang H, Shen B and Lanza GM: Atherosclerotic neovasculature MR imaging with mixed manganese-gadolinium nanocolloids in hyperlipidemic rabbits. Nanomedicine 11: 569-578, 2015.

17. Schinkel AF, van den Oord SC, van der Steen AF, van Laar JA and Sijbrands EJ: Utility of contrast-enhanced ultrasound for the assessment of the carotid artery wall in patients with Takayasu or giant cell arteritis. Eur Heart J Cardiovasc Imaging 15: 541-546, 2014.

18. Romero JM, Pizzolato R, Atkinson W, Meader A, Jaimes C, Lamuraglia G, Jaff MR, Buonanno F, Delgado Almandoz J and Gonzalez RG: Vasa vasorum enhancement on computerized tomographic angiography correlates with symptomatic patients with 50 to $70 \%$ carotid artery stenosis. Stroke 44: 3344-3349, 2013.

19. Hrkach J, Von Hoff D, Mukkaram Ali M, Andrianova E, Auer J, Campbell T, De Witt D, Figa M, Figueiredo M, Horhota A, et al: Preclinical development and clinical translation of a PSMA-targeted docetaxel nanoparticle with a differentiated pharmacological profile. Sci Transl Med 4: 128ra39, 2012

20. Pisani E, Tsapis N, Galaz B, Santin M, Berti R, Taulier N, Kurtisovski E, Lucidarme O, Ourevitch M, Doan BT, et al: Perfluorooctyl Bromide Polymeric Capsules as Dual Contrast Agents for Ultrasonography and Magnetic Resonance Imaging. Adv Funct Mater 18: 2963-2971, 2008.

21. Ghosh D, Lee Y, Thomas S, Kohli AG, Yun DS, Belcher AM and Kelly KA: M13-templated magnetic nanoparticles for targeted in vivo imaging of prostate cancer. Nat Nanotechnol 7: 677-682, 2012.

22. Li S, Ma Y, Yue X, Cao Z and Dai Z: One-pot construction of doxorubicin conjugated magnetic silica nanoparticles. New J Chem 33: 2414-2418, 2009.

23. Liu J, Li J, Rosol TJ, Pan X and Voorhees JL: Biodegradable nanoparticles for targeted ultrasound imaging of breast cancer cells in vitro. Phys Med Biol 52: 4739-4747, 2007.

24. Hermanson GT: Bioconjugate Techniques, I. Academic Press, London, pp169-172, 1996.

25. Tian C, Zhang R, Ye X, Zhang C, Jin X, Yamori Y, Hao L, Sun X and Ying C: Resveratrol ameliorates high-glucose-induced hyperpermeability mediated by caveolae via VEGF/KDR pathway. Genes Nutr 8: 231-239, 2013

26. Fonseca FA, Paiva TB, Silva EG, Ihara SS, Kasinski N, Martinez TL and Filho EE: Dietary magnesium improves endothelial dependent relaxation of balloon injured arteries in rats. Atherosclerosis 139: 237-242, 1998

27. Yan F, Yang W, Li X, Liu H, Nan X, Xie L, Zhou D, Xie G, Wu J, Qiu B, et al: Magnetic resonance imaging of atherosclerosis using CD81-targeted microparticles of iron oxide in mice. Biomed Res Int 2015: 758616, 2015 .
28. Guo S, Shen S, Wang J, Wang H, Li M, Liu Y, Hou F, Liao Y and Bin J: Detection of high-risk atherosclerotic plaques with ultrasound molecular imaging of glycoprotein IIb/IIIa receptor on activated platelets. Theranostics 5: 418-430, 2015.

29. Reimer P and Balzer T: Ferucarbotran (Resovist): A new clinically approved RES-specific contrast agent for contrast-enhanced MRI of the liver: Properties, clinical development, and applications. Eur Radiol 13: 1266-1276, 2003.

30. Wiesner P, Tafelmeier M, Chittka D, Choi SH, Zhang L, Byun YS, Almazan F, Yang X, Iqbal N, Chowdhury P, et al: MCP-1 binds to oxidized LDL and is carried by lipoprotein(a) in human plasma. J Lipid Res 54: 1877-1883, 2013.

31. Hsu WY, Chao YW, Tsai YL, Lien CC, Chang CF, Deng MC, Ho LT, Kwok CF and Juan CC: Resistin induces monocyte-endothelial cell adhesion by increasing ICAM-1 and VCAM-1 expression in endothelial cells via p38MAPK-dependent pathway. J Cell Physiol 226: 2181-2188, 2011.

32. Fotis L, Agrogiannis G, Vlachos IS, Pantopoulou A, Margoni A, Kostaki M, Verikokos C, Tzivras D, Mikhailidis DP and Perrea D: Intercellular adhesion molecule (ICAM)-1 and vascular cell adhesion molecule (VCAM)-1 at the early stages of atherosclerosis in a rat model. In Vivo 26: 243-250, 2012.

33. Deyama J, Nakamura T, Takishima I, Fujioka D, Kawabata K, Obata JE, Watanabe K, Watanabe Y, Saito Y, Mishina H and Kugiyama K: Contrast-enhanced ultrasound imaging of carotid plaque neovascularization is useful for identifying high-risk patients with coronary artery disease. Circ J 77: 1499-1507, 2013.

34. Li X, Bauer W, Israel I, Kreissl MC, Weirather J, Richter D, Bauer E, Herold V, Jakob P, Buck A, et al: Targeting P-selectin by gallium-68-labeled fucoidan positron emission tomography for noninvasive characterization of vulnerable plaques: Correlation with in vivo 17.6T MRI. Arterioscler Thromb Vasc Biol 34: 1661-1667, 2014.

35. Daeichin V, Kooiman K, Skachkov I, Bosch JG, Theelen TL, Steiger K, Needles A, Janssen BJ, Daemen MJ, van der Steen AF, et al: Quantification of Endothelial $\alpha v \beta 3$ expression with high-frequency ultrasound and targeted Microbubbles: In vitro and In vivo Studies. Ultrasound Med Biol 42: 2283-2293, 2016.

36. Qi CM, Du L, Wu WH, Li DY, Hao J, Gong L, Deng L, Zhang T, Zhang C and Zhang Y: Detection of Vulnerable Atherosclerotic Plaques in Experimental Atherosclerosis with the USPIO-Enhanced MRI. Cell Biochem Biophys 73: 331-337, 2015.

37. Wang Y, Chen J, Yang B, Qiao H, Gao L, Su T, Ma S, Zhang X, Li X, Liu G, et al: In vivo MR and Fluorescence Dual-modality Imaging of Atherosclerosis Characteristics in Mice Using Profilin-1 Targeted Magnetic Nanoparticles. Theranostics 6: 272-286, 2016

38. van Tilborg GA, Vucic E, Strijkers GJ, Cormode DP, Mani V, Skajaa T, Reutelingsperger CP, Fayad ZA, Mulder WJ and Nicolay K: Annexin A5-functionalized bimodal nanoparticles for MRI and fluorescence imaging of atherosclerotic plaques. Bioconjug Chem 21: 1794-1803,2010.

39. Gerber HP, McMurtrey A, Kowalski J, Yan M, Keyt BA, Dixit V and Ferrara N: Vascular endothelial growth factor regulates endothelial cell survival through the phosphatidylinositol 3'-kinase/Akt signal transduction pathway. Requirement for Flk-1/KDR activation. J Biol Chem 273: 30336-30343, 1998.

40. Arnott C, Punnia-Moorthy G, Tan J, Sadeghipour S, Bursill $\mathrm{C}$ and Patel S: The Vascular Endothelial Growth Factor Inhibitors Ranibizumab and Aflibercept Markedly Increase Expression of Atherosclerosis-Associated Inflammatory Mediators on Vascular Endothelial Cells. PLoS One 11: e0150688, 2016.

41. Kiessling F, Gaetjens J and Palmowski M: Application of molecular ultrasound for imaging integrin expression. Theranostics 1: 127-134, 2011.

42. Eckert MA, Vu PQ, Zhang K, Kang D, Ali MM, Xu C and Zhao W: Novel molecular and nanosensors for in vivo sensing. Theranostics 3: 583-594, 2013

43. Díaz-López R, Tsapis N and Fattal E: Liquid perfluorocarbons as contrast agents for ultrasonography and (19)F-MRI. Pharm Res 27: 1-16, 2010. 